Ann. Sci. forest., 1979, 36 (2), 125-149.

\title{
Les corrélations phénotypiques juvénile-adulte pour la densité du bois et la vigueur chez l'Epicéa
}

\author{
G. NEPVEU et Y. BIROT $\left(^{*}\right)$ \\ avec la collaboration de D. XEUXET \\ Station de Recherches sur la qualité des Bois, \\ Centre notional de Recherches forestières, I.N.R.A., \\ Chompenoux, 54280 Seichamps \\ - Station d'Amélioration des arbres forestiers, \\ Cenire de Recherches forestières d'Orléons, I.N.R.A., \\ Ardon, 45160 Olivet
}

\section{Résumé}

Après une importante revue bibliographique sur les corrélations juvénile-adulte pour divers caractères chez l'Epicéa, les auteurs ont évalué ces liaisons sur un peuplement d'une trentaine d'années en considérant les largeurs et les densités obtenues cerne par cerne par microdensitométrie.

Les corrélations entre cernes jeunes et adultes ont été étudiées pour ces deux caractéristiques en faisant varier l'âge des cernes jeunes ; il apparait que la prédiction de la densité est valable dès le jeune âge à la différence de l'accroissement en diamètre, en ne considérant qu'un seul cerne.

Une application de l'analyse de corrélations canoniques a été effectuée en utilisant des ensembles de cernes jeunes et adultes. L'intérêt de la méthode par rapport aux corrélations classiques est discuté.

La liaison défavorable densité-vigueur observée classiquement est confirmée. En revanche, l'antagonisme débourrement-densité n'apparait pas.

II semble que les individus les plus tardifs soient les plus sensibles aux attaques de Pristiphora abietina.

Jusqu'à une période relativement récente, le problème des corrélations juvénileadulte n'était abordé que de façon incidente. II faut remarquer également que lorsque des calculs étaient conduits sur divers matériels, on se souciait assez peu de leur interprétation génétique ; il est bien clair pourtant qu'une liaison n'a pas du tout la même signification si on l'étudie aux niveaux phénotypique, génołypique, environnemental, si l'on corrèle des moyennes de provenances dans le même milieu ou dans des expériences différentes... On se reportera à ce sujet à l'étude de Nepveu (1976) qui interprète les diverses corrélations calculées en matière d'étude de la qualité du bois.

Ces réserves étant faites, on trouvera dans le tableau 1 un certain nombre de résultats limités à l'essence qui nous intéresse et qui concernent des expériences et des matériels différents. On observera que la définition des états juvénile et adulte est très variable suivant les cas. 
TABLEAU 1

\begin{tabular}{|c|c|c|c|c|c|c|}
\hline \multicolumn{2}{|l|}{ Référence } & \multirow{2}{*}{$\begin{array}{l}\text { Caractère } \\
\text { hauteur }\end{array}$} & \multirow{2}{*}{$\begin{array}{l}\text { Etat adulte } \\
\text { plants de } 3 \text { à } 4 \text { ans }\end{array}$} & \multirow{2}{*}{\begin{tabular}{|c} 
Etat juvénile \\
les mèmes en pépinière
\end{tabular}} & \multirow{2}{*}{$\begin{array}{l}\text { Mèthode de calcul } \\
\begin{array}{c}- \\
\text { - }\end{array}\end{array}$} & \multirow{2}{*}{$\begin{array}{l}\text { Résultats et remarques } \\
\text { classement assez voisin aux } \\
\text { différents ages ( } 2 \text { essais) }\end{array}$} \\
\hline $\begin{array}{l}1931, \\
\text { Fabricius }\end{array}$ & (1) & & & & & \\
\hline $\begin{array}{l}1935, \\
\text { Bornebusch }\end{array}$ & (2) & hauteur & arbres de 20 ans & plants de 3 ans & - & $\begin{array}{l}\text { classement assez voisin des } \\
\text { croissances en hauteur entre } \\
\text { les deux âges extrêmes }\end{array}$ \\
\hline $\begin{array}{l}1949 . \\
\text { Munch }\end{array}$ & (3) & hauteur & arbres de 11 ans & plants de 3 ans & - & $\begin{array}{l}\text { classement assez voisin des } \\
\text { croissances en hauteur entre } \\
\text { les deux âges extrêmes }\end{array}$ \\
\hline $\begin{array}{l}1949 . \\
\text { Munch }\end{array}$ & (4) & hauteur & arbres de 7 et 19 ans & les mêmes en pépinière & - & $\begin{array}{l}\text { classement assez voisin aux } \\
\text { différents ages }\end{array}$ \\
\hline $\begin{array}{l}1949 . \\
\text { Munch }\end{array}$ & (5) & hauteur & $\begin{array}{l}\text { descendances maternelles } \\
\text { de } 11 \text { ans }\end{array}$ & $\begin{array}{l}\text { les mêmes à } 3 \text { ans en pé- } \\
\text { pinière }\end{array}$ & - & $\begin{array}{l}\text { pas de ressemblance entre les } \\
\text { classements }\end{array}$ \\
\hline $\begin{array}{l}1949 . \\
\text { Heikinheimo }\end{array}$ & & hauteur & arbres de 10 a 17 ans & les mêmes en pépinière & - & $\begin{array}{l}\text { classement assez voisin aux } \\
\text { différents âges (onze essais) }\end{array}$ \\
\hline $\begin{array}{l}1951 . \\
\text { Kiellander }\end{array}$ & (7) & hauteur & $\begin{array}{l}\text { provenances âgées de } \\
10 \text { ans }\end{array}$ & $\begin{array}{l}\text { provenances âgées de } \\
4 \text { ans }\end{array}$ & $\begin{array}{l}\text { corrélation entre moyen- } \\
\text { nes de provenances }\end{array}$ & $\begin{array}{l}r=0,85 \text { significatif pour l'é- } \\
\text { chantillon éfudié }\end{array}$ \\
\hline $\begin{array}{l}1952 . \\
\text { Rohmeder }\end{array}$ & (8) & hauteur & arbres de 11 ans & les mêmes en pépinière & - & $\begin{array}{l}\text { classement assez voisin aux } \\
\text { différents âges }\end{array}$ \\
\hline $\begin{array}{l}1957 . \\
\text { Petracic }\end{array}$ & (9) & hauteur & arbres de 15 ans & les mêmes en pépinière & - & $\begin{array}{l}\text { classement assez voisin aux } \\
\text { différents âges }\end{array}$ \\
\hline $\begin{array}{l}1957 \text {, } \\
\text { Rubner et } \\
\text { Schonbach }\end{array}$ & & houteur & arbres de 20 ans & arbres de 11 ans & - & $\begin{array}{l}\text { classement assez voisin des } \\
\text { croissances en hauteur entre } \\
\text { les deux âges extrêmes }\end{array}$ \\
\hline $\begin{array}{l}1958, \\
\text { Nilsson }\end{array}$ & (11) & $\begin{array}{l}\text { rapport hauteur dia- } \\
\text { métre (parents) et } \\
\text { hauteur (descen- } \\
\text { dants) }\end{array}$ & arbres adultes & $\begin{array}{l}\text { descendances maternel- } \\
\text { les âgées de } 10 \text { ans }\end{array}$ & $\begin{array}{l}\text { corrélation parent- } \\
\text { moyenne des descen- } \\
\text { dances }\end{array}$ & $\begin{array}{l}\text { liaison significative pour l'é- } \\
\text { chantillon étudié }(r=0,28)\end{array}$ \\
\hline $\begin{array}{l}1956 . \\
\text { Erichson }\end{array}$ & (12) & densité du bois & quinze arbres adultes & copies végétatives & corrélation ortet-ramet & $r=0,764^{* *}$ \\
\hline
\end{tabular}


TABLEAU 1 (suite)

\begin{tabular}{|c|c|c|c|c|c|}
\hline Référence & Caractère & Etat adulte & Etat juvénile & Méthode de calcul & Résultats et remarques \\
\hline $\begin{array}{l}\text { 1961, } \\
\text { Kleinschmit } \\
\\
\text { (13) }\end{array}$ & hauteur & $\begin{array}{l}\text { quatre-vingt-dix arbres } \\
\text { adultes }\end{array}$ & $\begin{array}{l}\text { leurs copies végétatives } \\
\text { après } 5 \text { saisons de végé- } \\
\text { tation }\end{array}$ & - & $\begin{array}{l}\text { le classement des boutures pour } \\
\text { la croissance est très différent } \\
\text { de celui des ortets }\end{array}$ \\
\hline $\begin{array}{l}\begin{array}{l}1962, \\
\text { Rohmeder ef al. } \\
\text { (14) }\end{array} \\
\end{array}$ & $\begin{array}{l}\text { résistance à } \mathrm{SO}_{2} \text { et } \\
\mathrm{HF}\end{array}$ & $\begin{array}{l}\text { trente-six arbres adultes } \\
\text { résistants ef dix-sept } \\
\text { non résistants }\end{array}$ & $\begin{array}{l}\text { copies végétatives des } \\
\text { arbres résistants et non } \\
\text { résistants (âge }<4 \text { ans) }\end{array}$ & - & $\begin{array}{l}\text { les ramets des ortets résistants } \\
\text { sont résistants }\end{array}$ \\
\hline $\begin{array}{l}\text { 1962, } \\
\text { Bouvarel }\end{array}$ & hauteur moyenne & $\begin{array}{l}\text { quinze provenances a } \\
4 \text { ans }\end{array}$ & $\begin{array}{l}\text { les mêmes à } 2 \text { ans avant } \\
\text { repiquage }\end{array}$ & $\begin{array}{l}\text { corrélation entre moyen- } \\
\text { nes de provenances }\end{array}$ & $\begin{array}{l}r=0,93^{* *}-r=0,77 \text { entre } \\
\text { hauteur aे } 2 \text { ans et accroisse- } \\
\text { ment de } 2 \text { aे } 4 \text { ans }\end{array}$ \\
\hline $\begin{array}{l}1964 . \\
\text { Burley, Mergen } \\
\text { et Yeatman (16) }\end{array}$ & débourrement & arbres adultes & $\begin{array}{l}\text { Copies végétatives en ser- } \\
\text { re }\end{array}$ & - & $\begin{array}{l}\text { les ramets des arbres précoces } \\
\text { en forết debourrent tốt en serre }\end{array}$ \\
\hline $\begin{array}{l}1968 . \\
\text { Dietrichson } \\
\text { (17) }\end{array}$ & hauteur & $\begin{array}{l}\text { huit provenances à } 12 \\
\text { ans }\end{array}$ & $\begin{array}{l}\text { les mêmes d̀ } 5 \text { ans ef d } \\
7 \text { ans }\end{array}$ & - & $\begin{array}{l}\text { classement bouleversé entre } \\
\text { l'état jeune et adulte à cause } \\
\text { de dommages climatiques }\end{array}$ \\
\hline $\begin{array}{l}\text { 1968, } \\
\text { Nanson }\end{array}$ & $\begin{array}{l}\text { hauteur moyenne } \\
\text { des arbres domi- } \\
\text { nants }\end{array}$ & $\begin{array}{l}\text { vingt provenances da } \\
25 \text { ans }\end{array}$ & les mêmes à 9 ans & $\begin{array}{l}\text { corrélation entre moyen- } \\
\text { nes de provenances }\end{array}$ & $\begin{array}{l}2 \text { dispositifs } A \text { et } B \text { : } \\
r=0,789 * * \text { (hauteurs } 9 \text { et } \\
25 \text { ans dispositif } A \text { ) } \\
r=0,858 * * \text { (hauteurs } 9 \text { et } \\
25 \text { ans dispositif } B \text { ) } \\
r=0,685 * * \text { (hauteur } 9 \text { ans } \\
\text { dispositif A et hauteur } 25 \text { ans } \\
\text { dispositif } B \text { ). } \\
r=0,810^{* *} \text { (hauteur } 9 \text { ans } \\
\text { dispositif B et hauteur } 25 \text { ans } \\
\text { dispositif } A \text { ) } \\
\text { (valeurs de } r \text { aussi élevées en } \\
\text { utilisant la hauteur da ans et } \\
\text { les accroissements entre } 9 \text { et } \\
25 \text { ans) }\end{array}$ \\
\hline $\begin{array}{l}1968, \\
\text { Nanson }\end{array}$ & hauteur moyenne & $\begin{array}{l}\text { quinze descendances a } \\
41 \text { ans }\end{array}$ & $\begin{array}{l}\text { les mêmes à } 3 \text { ans (pépi- } \\
\text { nière) } \\
\text { les mêmes à } 11 \text { ans }\end{array}$ & $\begin{array}{l}\text { corrélations entre } \\
\text { moyennes de descen- } \\
\text { dances } \\
\text { corrélations entre } \\
\text { moyennes de descen- } \\
\text { dances }\end{array}$ & $r=0,751 * *\left\{\begin{array}{l}\text { descendances } \\
\text { originaires } \\
\text { d'Autriche }\end{array}\right.$ \\
\hline
\end{tabular}


TABLEAU 1 (suite)

\begin{tabular}{|c|c|c|c|c|c|}
\hline Référence & Caractère & Etat adulte & Etat juvénile & Méthode de calcul & Résultats et remarques \\
\hline $\begin{array}{l}1968, \\
\text { Nanson }\end{array}$ & hauteur moyenne & $\begin{array}{l}\text { vingt-deux provenances } \\
\text { d } 2 \text { ans }\end{array}$ & les mêmes à 1 an & $\begin{array}{l}\text { corrélations entre } \\
\text { moyennes de provenan- } \\
\text { ces }\end{array}$ & $\begin{array}{l}r=0,956 * *-r=0,905 * * \\
\text { entre hauteur da } 1 \text { an et accrois- } \\
\text { sement entre } 1 \text { et } 2 \text { ans }\end{array}$ \\
\hline $\begin{array}{l}1969, \\
\text { Lacaze }\end{array}$ & débourrement & $\begin{array}{l}\text { cinquante-quatre arbres } \\
\text { adultes }\end{array}$ & $\begin{array}{l}\text { leurs descendances ma- } \\
\text { ternelles }\end{array}$ & $\begin{array}{l}\text { corrélation parent- } \\
\text { moyenne des descen- } \\
\text { dances }\end{array}$ & $\begin{array}{l}\text { très forte corrélation (Epicéas } \\
\text { du Jura français) }\end{array}$ \\
\hline $\begin{array}{l}1970, \\
\text { Lacaze }\end{array}$ & $\begin{array}{l}\text { débourrement } \\
\text { hauteur } \\
\text { hauteur }\end{array}$ & $\begin{array}{l}\text { à } 12 \text { ans } \\
\text { à } 12 \text { ans }\end{array}$ & $\begin{array}{l}\text { a } 2 \text { ans } \\
\text { à } 4 \text { ans }\end{array}$ & $\begin{array}{l}\text { corrélation entre } \\
\text { moyennes de provenan- } \\
\text { ces dans chaque dispo- } \\
\text { sitif } \\
\text { corrélation entre } \\
\text { moyennes de provenan- } \\
\text { ces dans chaque disposi- } \\
\text { sitif } \\
\text { corrélation entre } \\
\text { moyennes de provenan- } \\
\text { ces dans chaque disposi- } \\
\text { tif }\end{array}$ & $\begin{array}{l}0,94 * * \text { (I) } \\
0,92 * * \text { (II) } \\
0,90 * * \text { (III) } \\
-0,35 \text { NS (I) } ; 0,85 * \text { (II) } \\
0,69 * * \text { (III) } \\
-0,09 \text { NS (I) } ; 0,92 * * \text { (II) } \\
0,67 * * \text { (III) } \\
\text { (provenances françaises } \\
\text { - sauf } 1 \text { autrichienne - dans } \\
\text { dispositifs : } \\
\text { disp. I } 7 \text { provenances } \\
\text { disp. II }=13 \text { provenances } \\
\text { disp. III }=11 \text { provenances) }\end{array}$ \\
\hline $\begin{array}{l}1971, \\
\text { Lacaze et Arbez } \\
\text { (23) }\end{array}$ & hauteur & $\begin{array}{l}\text { plusieurs descendances } \\
\text { dans plusieurs prove- } \\
\text { nances d } 4 \text { ans }\end{array}$ & les mêmes à 3 ans & $\begin{array}{l}\text { corrélation génétique } \\
\text { intraprovenance }\end{array}$ & $\begin{array}{l}r=0,96 \text { à } 0,85 \text { suivant les pro- } \\
\text { venances (valeurs très signifi- } \\
\text { catives) } \\
\text { (provenances françaises) }\end{array}$ \\
\hline $\begin{array}{l}1971 . \\
\text { Nanson }\end{array}$ & $\begin{array}{l}\text { débourrement } \\
\text { hauteur } \\
\text { fréquence des pous- } \\
\text { ses d'Aoôt }\end{array}$ & $\begin{array}{l}\text { trente-et-une descendan- } \\
\text { ces maternelles à } 16 \text { ans } \\
\text { en plantation } \\
\text { les mềmes à } 7 \text { et } 15 \text { ans } \\
\text { en plantation } \\
\text { les mêmes à } 15 \text { ans }\end{array}$ & $\begin{array}{l}\text { les mêmes à } 5 \text { ans en } \\
\text { pépinière } \\
\text { les mêmes d̀ } 1 \text { et } 4 \text { ans } \\
\text { en pépinière } \\
\text { les mêmes à } 5 \text { ans en } \\
\text { pépinière }\end{array}$ & $\begin{array}{l}\text { corrélation entre } \\
\text { moyennes de descen- } \\
\text { dances } \\
\text { corrélation entre } \\
\text { moyennes de descen- } \\
\text { dances } \\
\text { corrélation entre } \\
\begin{array}{l}\text { moyennes de descen- } \\
\text { dances }\end{array}\end{array}$ & $\begin{array}{l}r=0,76 * \\
r=0,40 *(1 \text { an, } 7 \text { ans }) \\
r=0,39 *(1 \text { an, } 15 \text { ans }) \\
r=0,44 *(4 \text { ans, } 7 \text { ans) } \\
r=0,68 * * \text { (4 ans, } 15 \text { ans) } \\
r=0,15 \text { NS }\end{array}$ \\
\hline
\end{tabular}


TABLEAU 1 (suife)

\begin{tabular}{|c|c|c|c|c|c|}
\hline Référence & Caractère & Etat adulte & Etat juvénile & Méthode de calcul & Résultats et remarques \\
\hline $\begin{array}{l}1971, \\
\text { Nanson }\end{array}$ & angle de branche & $\begin{array}{l}\text { seize descendances ma- } \\
\text { ternelles à } 11 \text { ans } \\
\text { les mêmes à } 5 \text { ans }\end{array}$ & $\begin{array}{l}\text { les mêmes à } 8 \text { ans } \\
\text { les mêmes à } 3 \text { ans }\end{array}$ & $\begin{array}{l}\text { corrélation entre } \\
\text { moyennes de descen- } \\
\text { dances } \\
\text { corrélation entre } \\
\text { moyennes de descen- } \\
\text { dances }\end{array}$ & $\begin{array}{l}r=0,68 * \\
r=0,62 *\end{array}$ \\
\hline $\begin{array}{l}1971 \text {, } \\
\text { Rohmeder (26) }\end{array}$ & $\begin{array}{l}\text { épaisseur de l'écor- } \\
\text { ce }\end{array}$ & arbres adultes & $\begin{array}{l}\text { copies végétatives de } 3 \\
\text { ans }\end{array}$ & - & $\begin{array}{l}\text { les ramets des arbres d̀ écorce } \\
\text { épaisse ont une écorce épaisse }\end{array}$ \\
\hline $\begin{array}{l}1972, \\
\text { Von Reck }\end{array}$ & débourrement & seize arbres adultes & $\begin{array}{l}\text { vingt-deux descendances } \\
\text { de } 5 \text { a } 6 \text { ans provenant } \\
\text { de divers croisements } \\
\text { entre les arbres adultes }\end{array}$ & $\begin{array}{l}\text { corrélation parents-des- } \\
\text { cendants }\end{array}$ & $r=0.85$ \\
\hline $\begin{array}{l}1972, \\
\text { Kowalski et } \\
\text { Wloczewski }\end{array}$ & hauteur & $\begin{array}{l}\text { neuf provenances a } 14 \\
\text { ans }\end{array}$ & $\begin{array}{l}\text { les mêmes à } 1,3,4,6,7 \text {. } \\
8 \text { ans }\end{array}$ & $\begin{array}{l}\text { corrélation entre moyen- } \\
\text { nes de provenances }\end{array}$ & $\begin{array}{l}r=0,233 \text { NS }(1 \text { an- } 14 \text { ans) } \\
r=0,337 \text { NS }(3 \text { ans- } 14 \text { ans) } \\
r=0,399 \text { NS (4 ans-14 ans) } \\
r=0,488 \text { NS ( } 6 \text { ans-14 ans) } \\
r=0,768 * \text { ( } 7 \text { ans- } 14 \text { ans) } \\
r=0,867 * \text { ( } 8 \text { ans-14 ans) } \\
\text { (provenances polonaises }-r e ́- \\
\text { sultats très voisins en consi- } \\
\text { dérant les accroissements cou- } \\
\text { rants) }\end{array}$ \\
\hline $\begin{array}{l}\text { 1973, } \\
\text { Gislerud }\end{array}$ & densité du bois & arbres d'élite & $\begin{array}{l}\text { copies végétatives de } 6 \text { à } \\
13 \text { ans }\end{array}$ & corrélation ortet-ramet & $r=0,51 \cdots(47 d d l)$ \\
\hline $\begin{array}{l}1973 \text {, } \\
\text { Baldwin, Eliason } \\
\text { et Carison }\end{array}$ & hauteur & $\begin{array}{l}\text { vingt-deux d trente-six } \\
\text { provenances de } 8 \text { da } \\
25 \text { ans }\end{array}$ & les mêmes de 1 à 4 ans & $\begin{array}{l}\text { corrélation entre moyen. } \\
\text { nes de provenances }\end{array}$ & $\begin{array}{l}r=0,706 * \text { ( } 1 \text { et } 4 \text { ans) } 32 \text { ddl } \\
r=0,481 *(4 \text { et } 8 \text { ans) } 22 \text { ddl } \\
r=0,303 *(4 \text { et } 20 \text { ans) } 34 \text { ddl } \\
r=0,756 * \text { (4 et } 25 \text { ans) } 25 \text { ddl }\end{array}$ \\
\hline $\begin{array}{l}1973 . \\
\text { Lewis et Lines } \\
\text { (31) }\end{array}$ & débourrement & $\begin{array}{l}\text { vingt-deux provenances } \\
\text { après } 3 \text { ans de planta- } \\
\text { lion }\end{array}$ & $\begin{array}{l}\text { les mèmes en pépinière à } \\
4 \text { ans }\end{array}$ & $\begin{array}{l}\text { corrélation entre moyen- } \\
\text { nes de provenances }\end{array}$ & $r=0.89 * *$ \\
\hline $\begin{array}{l}1975, \\
\text { Badan et Jeantet } \\
(32)\end{array}$ & hauteur & $\begin{array}{l}\text { trente-sept provenances à } \\
8 \text { ans }\end{array}$ & $\begin{array}{l}\text { les mêmes à } 2 \text { ans avant } \\
\text { repiquage }\end{array}$ & - & $\begin{array}{l}\text { classements très proches dans } \\
\text { les deux cas (provenances } \\
\text { suisses) }\end{array}$ \\
\hline
\end{tabular}


TABLEAU 1 (suite)

\begin{tabular}{|c|c|c|c|c|c|}
\hline Référence & Caractère & Etat adulte & Etat juvénile & Méthode de calcul & Résultats et remarques \\
\hline $\begin{array}{l}\text { 1975, } \\
\text { Teissier du Cros }\end{array}$ & $\begin{array}{l}\text { angle de branche } \\
\text { diamètre de la plus } \\
\text { grosse branche } \\
\text { vigueur } \\
\text { débourrement }\end{array}$ & $\begin{array}{l}\text { seize arbres adultes avec } \\
\text { témoin } \\
\text { seize arbres adultes avec } \\
\text { témoin } \\
\text { seize arbres adultes avec } \\
\text { témoin } \\
\text { seize arbres adultes avec } \\
\text { témoin }\end{array}$ & $\begin{array}{l}\text { leurs descendances ma- } \\
\text { ternelles d̀ } 11 \text { ans } \\
\text { leurs descendances ma- } \\
\text { ternelles à } 11 \text { ans } \\
\text { leurs descendances ma- } \\
\text { ternelles d̀ } 11 \text { ans } \\
\text { leurs descendances ma- } \\
\text { ternelles à } 11 \text { ans }\end{array}$ & $\begin{array}{c}- \\
\text { corrélations parents- } \\
\text { moyennes des descen- } \\
\text { dances }\end{array}$ & $\begin{array}{l}\text { descendances des arbres }+ \text { à } \\
\text { angle de branche plus fort que } \\
\text { celui des témoins } \\
\text { aucun gain par rapport au } \\
\text { témoin } \\
\text { aucun gain par rapport au } \\
\text { témoin } \\
r=0,72 * \text { à } 0,78 * \text { suivant } \\
\text { l'année de notation de l'arbre } \\
\text { mère } \\
\text { (épicéas vosgiens sélection- } \\
\text { nés pour leur vigueur leurs } \\
\text { branches courtes et horizon- } \\
\text { tales) }\end{array}$ \\
\hline $\begin{array}{l}\text { 1975, } \\
\text { Teissier du Cros } \\
\text { (34) }\end{array}$ & débourrement & $\begin{array}{l}\text { dix descendances mater- } \\
\text { nelles d } 9 \text { ans }\end{array}$ & $\begin{array}{l}\text { les mềmes à } 4 \text { ans (pépi- } \\
\text { nière) }\end{array}$ & $\begin{array}{l}\text { corrélation entre moyen- } \\
\text { nes des descendances }\end{array}$ & $\begin{array}{l}r=0,62 * \text { (descendances d'épi- } \\
\text { céas vosgiens se retrouvant } \\
\text { dans le dispositif précédent) }\end{array}$ \\
\hline $\begin{array}{l}1976 \text {, } \\
\begin{array}{l}\text { Weisgerber } \\
\text { et al. }\end{array}\end{array}$ & hauteur & $\begin{array}{l}\text { quatre cent soixante-trei- } \\
\text { ze provenances d } 8 \text { ans } \\
\text { en plantation }\end{array}$ & $\begin{array}{l}\text { les mêmes d̀ } 4 \text { ans sur } \\
\text { planches de repiquage }\end{array}$ & - & $\begin{array}{l}\text { classements très proches dans } \\
\text { les deux cas (plusieurs disposi- } \\
\text { tifs) }\end{array}$ \\
\hline $\begin{array}{l}1977 . \\
\text { Parde }\end{array}$ & hauteur & $\begin{array}{l}\text { provenances d'Europe } \\
\text { Centrale à } 17 \text { ans }\end{array}$ & les mêmes à 8 ans & - & $\begin{array}{l}\text { la meilleure provenance d̀ } 8 \text { ans } \\
\text { est la moins bonne da } 17 \text { ans. } \\
\text { La meilleure da } 17 \text { ans est } 7^{6} \text { dे } \\
8 \text { ans. } \\
\text { (dispositif installé au Canada) }\end{array}$ \\
\hline $\begin{array}{l}\text { non publié } \\
\text { Wellendorf et } \\
\text { Olesen } \quad \text { (37) }\end{array}$ & densité du bois & arbres de 33 ans & $\begin{array}{l}\text { copies végétatives de } 18 \\
\text { ans }\end{array}$ & corrélation ortet-ramet & $r=0,56 *(13 \mathrm{ddl})$ \\
\hline
\end{tabular}

Un nombre important de références sont empruntées à l'ouyrage de NANSON (1968) : les 11 premiéres et la 14e. Dans de nombreux cas, des calculs ont été effectués par nous-mêmes à partir des données de bases présentes dans les articles. 
On peut, caractère par caractère, faire les observations suivantes qui sont naturellement à considérer avec circonspection lorsque les références sont peu nombreuses:

- débourrement : tous les auteurs notent une excellente liaison juvénile-adulte (huit références sur huit) ;

— densité du bois : bonne stabilité (trois références sur trois) ;

- hauteur : les liaisons sont relativement bonnes, moins étroites cependant que pour les deux caractères précédents (vingt et une références sur vingt-sept) :

- résistance aux gaz polluants : bonne stabilité génotypique (une référence) ;

- fréquence des pousses d'aoôt : pas de stabilité (une référence) ;

- épaisseur de l'écorce : bonne stabilité (une référence) ;

- angle de branche : assez bonne stabilité (deux références) ;

- diamètre de la plus grosse branche : pas de stabilité (une référence).

Le travail dont nous présentons maintenant les résultats a pour but de préciser les liaisons phénotypiques juvénile-adulte chez l'épicéa pour la densité du bois et l'accroissement sur le diamètre. Nous en soulignerons l'intérêt pratique, au plan sylvicole notamment.

Il s'agit en fait d'une étude préliminaire faisant partie d'un projet plus vaste concernant un test clonal dont les résultats seront publiés prochainement. Incidemment, nous discuterons de quelques corrélations entre caractères.

\section{1. - Matériel et méthodes}

\section{1. - Matériel végéfal et dispositifs expérimentaux}

Onze descendances maternelles (pollinisation libre) ont été récoltées sur des arbres mères appartenant à un peuplement artificiel proche de Gdansk (Pologne) dont l'origine serait lithuanienne, région de provenances intéressante pour les conditions françaises de reboisement. Les arbres mères auraient été sélectionnés avant la guerre par Nikolai sur des critères qui ne sont pas connus; certains de ces arbres retrouvés en 1961 ne semblent pas, au moins pour la vigueur, présenter une supériorité phénotypique par rapport à leurs voisins (Bouvarel, communication personnelle). Si la sélection opérée a vraisemblablement peu biaisé la variabilité génétique existante, plus gênant est le fait que les arbres mères appartiennent à des bouquets différents répartis au milieu de peuplements d'une autre espèce ; ceci biaiserait toutes les estimations de certains paramètres génétiques qui reposent en particulier sur une hypothèse de panmixie.

Après élevage en pépinière, deux tests de descendances ont été nstallés dans les arboretums d'Amance et de La Sivrite proches de Nancy; leurs caractéristiques principales sont rappelées au tableau 2 *. En 1957, Bouvarel et Lemoine ont sélectionné dans les tests de descendances cinquante et un individus au total répartis dans toutes les familles, mais en nombre inégal (de un à sept suivant les descendances) sur les critères suivants : hauteur totale et, dans une moindre mesure, circonférence, résistance aux némates (Pristiphora abietina) à Amance ; bien qu'il ait été observé, le

* L'absence de répétitions constitue la faiblesse majeure de ce dispositif. 
débourrement végétatif n'a pas été retenu. L'intensité de sélection varie suivant les descendances de 3 à 22 p. 100 ; en moyenne elle est de 10 p. 100.

TABLEAU 2

Description des dispositifs expérimentoux

Description of experimental plots

\begin{tabular}{ccccc}
\hline \multicolumn{1}{c}{ Lieu } & $\begin{array}{c}\text { Année de } \\
\text { plantation }\end{array}$ & Espacement & $\begin{array}{c}\text { Nombre de } \\
\text { descendances } \\
\text { (ou de clones) }\end{array}$ & $\begin{array}{c}\text { Dispositif } \\
\text { expérimental }\end{array}$ \\
\hline Amance........ & 1943 & $2 \mathrm{~m} \times 2 \mathrm{~m}$ & 8 & $\begin{array}{l}20 \text { à } 45 \text { arbres } \\
\text { par descendance }\end{array}$ \\
La Sivrite ...... & 1943 & $1,5 \mathrm{~m} \times 1,5 \mathrm{~m}$ & 4 ( $\left.^{1}\right)$ & $\begin{array}{l}40 \text { à } 120 \text { arbres } \\
\text { par descendance }\end{array}$ \\
\hline
\end{tabular}

(') 2 descendances sont communes à chaque station.

\section{2. - Caractères mesurés ou observés}

Le tableau 3 récapitule les caractères observés ef l'échantillonnage correspondant. On remarque qu'une grande partie des mesures n'a été réalisée que sur les individus sélectionnés.

On peut préciser les points suivants concernant la détermination des infradensités et des densités :

Les infradensités ont été obtenues sur des carottes de sondage prises à 1,30 m de hauteur dont on a découpé des tronçons correspondant aux âges qui nous intéressaient. L'âge du cerne est déterminé en notant sa position par rapport à la moelle (le cerne de un an est le premier accroissement voisin de la moelle) de sorte que, la croissance en hauteur des arbres étant variable et malgré la simultanéité d'installation de tous les plants, les cernes indexés la même année n'ont pas forcément le même âge.

L'infradensité qui est le rapport $P_{\mathrm{o}} / \mathrm{V}_{\mathrm{s}}$ (poids anhydre rapporté au volume saturé) est obtenue grâce aux poids anhydre $P_{0}$ et saturé $P_{8}$ du même échantillon en utilisant la formule de Keylwerth (1954):

$$
\frac{P_{0}}{V_{s}}=\frac{1}{\frac{P_{s}}{P_{0}}-0,347} .
$$

Un problème se posait pour le prélèvement des carottes de sondage à La Sivrite du fait des écartements différents entre les lignes de la même famille $(1,50 \mathrm{~m})$ et entre celles correspondant à deux familles différentes $(3 \mathrm{~m})$. On pouvait s'attendre à observer une anisotropie de gradient perpendiculaire aux lignes; des phénomènes de ce genre sont très fréquemment cités dans la littérature (Polge et Illy, 1967 ; Janin, 1973 ; Thoby, 1975). Pour cette raison, dans cette parcelle, les échantillons ont été prélevés perpendiculairement aux lignes; les données obtenues sur les éprouvettes de La Sivrite sont les moyennes des valeurs correspondant aux deux demi-carottes (ce, pour 
TABLEAU 3

Coractères observès dans les diffërents dispositiff ; échantillonnage

Characteristics observed in the two experimental plots ; sampling

\begin{tabular}{|c|c|c|c|c|c|}
\hline & Caractères & Années de mesure & Echantillons & $\begin{array}{l}\text { Nota- } \\
\text { tions }\end{array}$ & Observations \\
\hline $\begin{array}{c}\text { Test de } \\
\text { descendances } \\
\text { Amance }\end{array}$ & $\begin{array}{l}\text { - infradensité } \\
\text { - densité et largeur cerne } \\
\text { par cerne } \\
\text { - circonférence } \\
\text { - hauteur } \\
\text { - dégâts de némates } \\
\text { (Pristiphora abietina) } \\
\text { - dégâts de gelée } \\
\text { - note de débourrement } \\
\text { - note de débourrement } \\
\text { - hauteur } \\
\text { - circonférence }\end{array}$ & $\begin{array}{l}\text { toutes années de la moelle à } \\
\text { l'écorce (vingt-cinq cernes env.) } \\
1956-1976 \\
1956 \\
1952 \\
1953 \\
1955 \\
1951-1954 \\
1949 \text { d } 1954-1968 \\
1957 / 59 / 61 / 68\end{array}$ & $\begin{array}{l}\text { totalité des arbres } \\
\text { arbres sélectionnés } \\
\text { totalité des arbres } \\
\text { totalité des arbres } \\
\text { totalité des arbres } \\
\text { totalité des arbres } \\
\text { totalité des arbres } \\
\text { arbres sélectionnés } \\
\text { arbres sélectionnés } \\
\text { arbres sélectionnés }\end{array}$ & $\begin{array}{l}- \\
- \\
- \\
0 \text { d } 5 \\
0 \text { à } 5 \\
0 \text { à } 5 \\
0 \text { à } 5 \\
- \\
-\end{array}$ & $\begin{array}{l}\text { carottes de sondage prélevées en } 1976 \\
\text { par microdensitométrie sur carottes de } \\
\text { sondage } \\
\text { par microdensitométrie sur carottes de } \\
\text { sondage } \\
\text { par microdensitométrie sur carottes de } \\
\text { sondage } \\
\text { note croissant avec l'importance des dé- } \\
\text { gâts } \\
\text { note croissant avec l'importance des dé- } \\
\text { gâts } \\
\text { note croissant avec la précocité du sujet } \\
\text { note croissant avec la précocité du sujet } \\
-\end{array}$ \\
\hline $\begin{array}{c}\text { Test de } \\
\text { descendances } \\
\text { La Sivrite }\end{array}$ & $\begin{array}{l}\text { - densité et largeur cerne } \\
\text { par cerne } \\
\text { - circonférence } \\
\text { - hauteur } \\
\text { - dégâts de gelée } \\
\text { - note de débourrement }\end{array}$ & $\begin{array}{c}\text { toutes années de la moelle à } \\
\text { l'écorce (vingt-cinq cernes env.) } \\
1976 \\
1956 \\
1953 \\
1953\end{array}$ & $\begin{array}{l}\text { arbres sélectionnés et } \\
\text { échantillons aléatoi- } \\
\text { res de trente arbres } \\
\text { par famille } \\
\text { arbres sélectionnés } \\
\text { totalité des arbres } \\
\text { totalité des arbres } \\
\text { totalité des arbres } \\
\text { totalité des arbres }\end{array}$ & $\begin{array}{l}- \\
\overline{0} \overline{\text { a } 5} \\
0 \text { d̀ } 5\end{array}$ & $\begin{array}{l}\text { par microdensitométrie sur carottes de } \\
\text { sondage } \\
\text { note croissant avec l'importance des dé- } \\
\text { gâts } \\
\text { note croissant avec la précocité du sujet }\end{array}$ \\
\hline
\end{tabular}


les infradensités observées sur des tranches de quatre années, mais également pour les variables notées au densitomètre : voir plus avant).

A Amance, la plantation ayant été effectuée à $2 \mathrm{~m}$ en tous sens, et étant en outre bien profégée des vents dominants par les peuplements avoisinants, seule une demicarotte a été prise sur la face nord des arbres, parallèlement aux lignes.

Par la suite, ayant besoin d'apprécier l'infradensité en 1957, date de la sélection, et également en 1976, on a prélevé de nouvelles carottes traversant l'arbre de part en part et parallèlement aux lignes (direction neutre) tant à Amance qu'da La Sivrife; ceci nous permettait d'éviter de mêler dans nos caiculs des chiffres qui représentent une moyenne de deux données (La Sivrite) ou une donnée simple (Amance). Par « infradensité 1956 », on doit donc entendre celle de la carotte entière ; par « infradensité 1976 » celle du même échantillon privé à ses deux extrémités du bois formé de 1957 à 1976.

Les densités et les largeurs des accroissements annuels ont été obtenues par analyse densitométrique selon la méthode établie par Polge (1966) ; les carottes, après avoir été amenées à un taux d'humidité de 10 p. 100 environ, ont été radiographiées dans les conditions expérimentales qui suivent :

- distance émetteur-éprouvette : $2,50 \mathrm{~m}$,

- tension : $15 \mathrm{kV}$,

- intensité : $20 \mathrm{~mA}$,

- temps d'exposition : $4^{\prime} 30^{\prime \prime}$.

On se reportera au travail de Thoby $(1975$, op. cif.) qui explique la détermination des densités moyennes de cernes à partir des courbes densitométriques.

\section{2. - Résultats et discussion}

L'absence de dispositif statistique, la présence des familles dans deux stations différentes ne permettent pas de séparer d'éventuels effets génétiques de ceux du milieu. On éfudiera seulement les liaisons juvénile-adulte pour la densité ef la largeur de cerne, ainsi que les relations largeur-densité en considérant les corrélations intra-familles $r_{\mathrm{e}}$ qui sont débarrassées d'un effet «famille » difficile à caractériser (ce qui permettra de traiter ensemble les données provenant d'Amance et de La Sivrite).

Les calculs réalisés ont été des analyses de variance-covariance à un facteur, l'effet contrôlé étant celui de la descendance.

On sait que les variances et covariances intra-familles ainsi calculées sont très voisines des variances et covariances phénotypiques d'où l'assimilation de $r_{e}$ à un coefficient de corrélation phénotypique. En effet, dans le cas de familles de demifrères - en admettant il est vrai certaines hypothèses qui ne sont peut-être pas toutes satisfaites ici (panmixie notamment) - on a :

$$
\sigma_{\mathrm{e}}^{2}=\frac{3}{4} \mathrm{~V}_{\mathrm{A}}+\mathrm{V}_{\mathrm{D}}+\mathrm{V}_{\mathrm{B}}
$$

On voit que $\sigma_{e}^{2}$ est très proche de la variance phénotypique égale par définition à $V_{A}+V_{D}+V_{E}$ (avec $V_{A}, V_{D}$ et $V_{E}$ respectivement variances des effets génétiques additifs, génétiques non additifs et dus à l'environnement). 
2.1. - Coractères mesurés âge par âge au densitomètre : densité ef largeur de cerne

Les cernes sont classés suivant leur âge à partir de la moelle. On notera respectivement $D_{n}$ et $L_{n}$ les densités et largeur du cerne d'âge $n$. Pour les deux parcelles (Amance et La Sivrife), on a étudié quarante-cinq arbres appartenant à dix familles dont les caractéristiques $D_{n}$ et $L_{n}$ ont été relevées de 6 à 26 ans (douze à La Sivrite, trente-trois à Amance).

Pour des raisons techniques (essentiellement parce que la carotte de sondage ne passait pas exactement au çeur de l'arbre), $D_{n}$ et $L_{n}$ n'ont pas été relevées dès l'âge 1 ; on a observé cependant $D_{3}$ et $L_{3}$ sur vingt-deux individus (groupés en huit familles), $D_{4}$ et $L_{4}$ sur trente-sept arbres (dix familles), $D_{5}$ ef $L_{5}$ sur quarante-trois (dix familles également).

On a déjà fait remarquer que, du fait de la croissance inégale des arbres en hauteur, le bois d'un âge donné n'est pas formé la même année pour tous les individus, même s'ils ont été plantés simultanément. Par conséquent, il est possible que, par suite de l'influence plus ou moins forte des variations climatiques annuelles, les corrélations

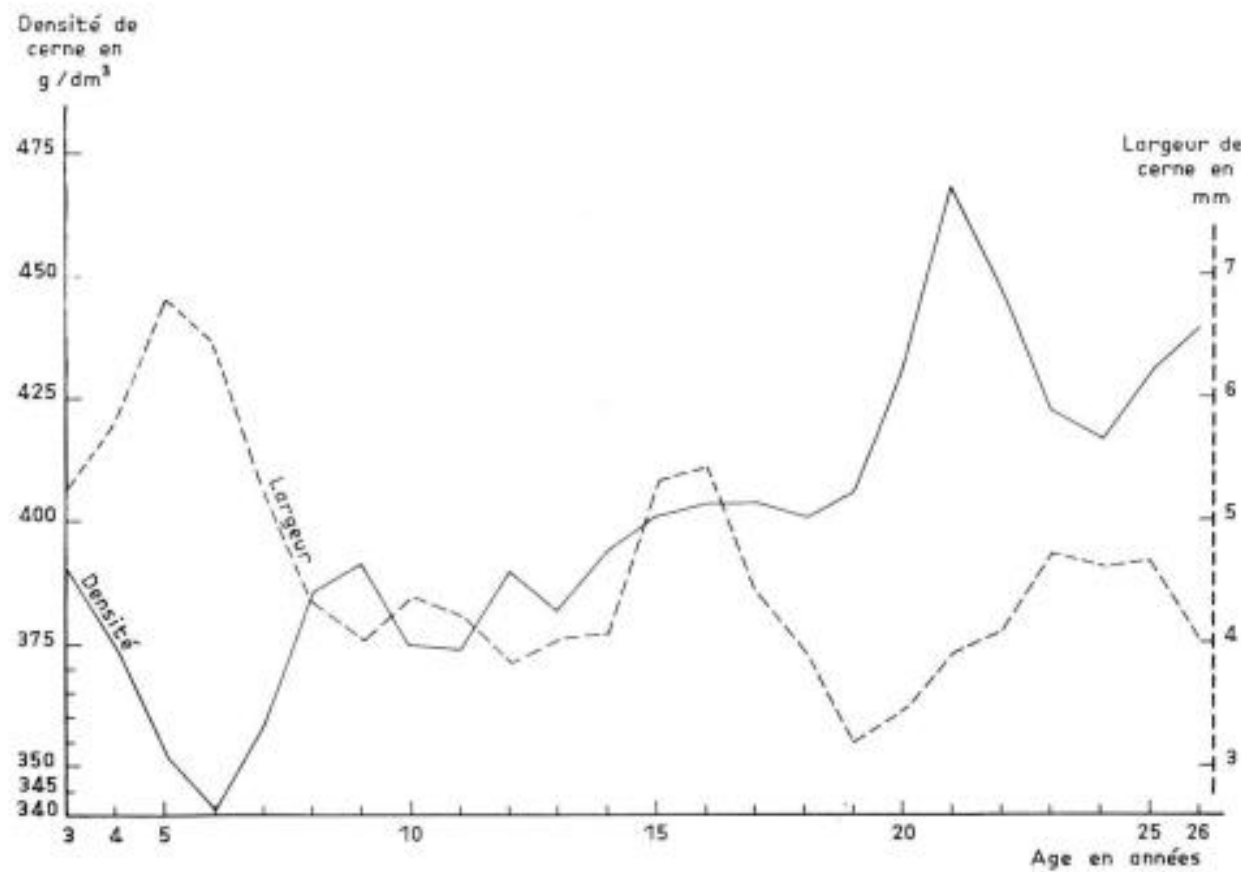

FIG. 1. - Evolution avec l'äge de la densité ef de lo largeur de cerne. Moyennes générales portant sur les arbres sélectionnés d'Amance et de La Sivrite.

-3 ans : 22 individus,

- 4 ans : 37 individus.

-5 ans : 43 individus,

- 6 à 26 ans : 45 individus.

Variation of ring wood densify and ring width according to age. General mean calculated for selected Irees in Amance and Lo Sivrite. 
calculées soient plus faibles que celles obtenues avec des carottes de sondage présentant le même nombre d'accroissements depuis la moelle ; ceci contribue à donner à nos résultats une portée plus générale.

\subsection{Evolution de la densifé et de la largeur de cerne avec l'âge.}

La figure 1 montre une augmentation assez régulière de la densité avec l'âge quí se poursuit encore vers 20-25 ans, après avoir chuté jusqu'à l'âge de six années quí correspond sans doute à la limite du bois juvénile. Ce phénomène a déjà été observé par Polge (1964) sur la même essence. La largeur du cerne, après une baisse vers 7 à 8 ans, ne semble pas manifester de tendances particulières à un âge plus avancé. Notons que le pic observé vers 15-16 ans correspond peut-être à l'effet de l'éclaircie de 1959 à Amance (cette année-là, les tiges présentaient entre douze et quatorze cernes).

\subsection{Liaisons densité-largeur.}

La figure 2 montre que, malgré une « tendance » négative des liaisons entre $D$ ef $L$, seules quatre valeurs sont statistiquement non nulles aux ages suivants : 8,9 , 22 et 23 ans. Ceci semblerait contradictoire, d'une part avec l'opinion générale des forestiers et chercheurs, d'autre part avec certains résultats du tableau 4 qui indiquent

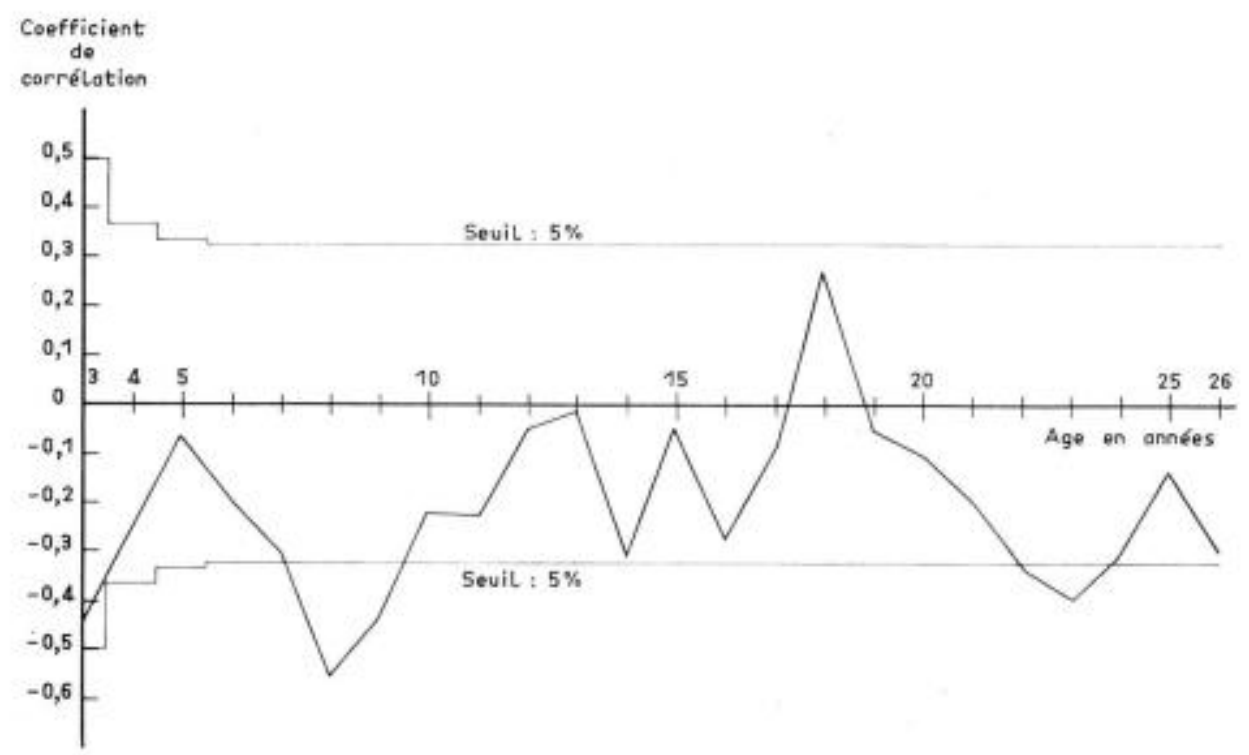

FIG. 2. - Liaisons suivant les âges entre densité et largeur de cerne. Arbres sélectionnès d'Amance et de La Sivrite.

Sevils de signification à 5 p. 100 :

- d 3 ans : 0,497 (14 ddl : 8 familles, 22 observations),

- à 4 ans : 0,367 (27 ddl : 10 familles, 37 observations),

- d 5 ans : 0,334 (33 ddl : 10 familles, 43 observations),

- de 6 da 26 ans : 0,325 (35 ddl : 10 familles, 45 observations).

Relationships between ring wood density and ring width in terms of age. Selected trees in Amance and La Sivrite. 
des liaisons infradensité - circonférence négatives en 1956 et 1976 et ce, aussi bien dans la population des ortets sélectionnés que dans celle de départ. En fait, ceci s'explique par la nature cumulative du caractère circonférence. Notons également qu'aucune évolution des valeurs observées ne se manifeste de façon systématique avec l'âge.

Nous signalerons toutefois au lecteur qu'il est nécessaire d'être circonspect dans l'interprétation des tests statistiques vis-à-vis d'hypothèses concernant une succession de coefficients de corrélation ; s'il est vrai par exemple que la corrélation largeur $x$ densité n'est pas statistiquement différente de zéro au seuil de 5 p. 100 à chacun des âges considérés (sauf 8, 9, 22 et 23 ans), on ne peut en déduire au niveau global, c'est-à-dire en considérant les âges dans leur ensemble, l'absence de liaison entre les deux caractères. Cette remarque vaudra pour les résultats des deux paragraphes suivants.

\subsection{Liaisons juvénile-adulte pour la largeur de cerne.}

Remarquons en préambule que le choix du caractère « largeur de cerne » n'est peut-être pas très satisfaisant pour le praticien qui est plus sensible à l'accroissement en surface terrière. La raison en est que les carottes de sondage ne passant pas toutes par le cœur de l'arbre, il était difficile, surtout dans le jeune âge, de calculer ce paramètre qui intègre non seulement la largeur $L_{n}$ du cerne d'âge $n$, mais aussi la distance de l'accroissement annuel au cœur de l'arbre, soit $L_{1}+L_{2}+\ldots+L_{n-1}$. On se rend compte également que ce procédé introduirait une liaison « mécanique » entre les largeurs de cernes dues à la prise en compte du terme $L_{1}+L_{2}+\ldots+L_{n-1}$.

Disposant de L pour vingt-quatre cernes (trois à 26 ans), on a calculé deux cent soixante-seize corrélations, certaines entre cernes voisins, d'autres entre cernes très éloignés. Nous en donnons dans la figure 3 un exemple.

II s'agit des corrélations de $\mathrm{L}_{26}$ avec $\mathrm{L}_{3}, \mathrm{~L}_{4}, \ldots, \mathrm{L}_{23}, \mathrm{~L}_{24}$ d'une part, et de $\mathrm{L}_{25}$ avec $\mathrm{L}_{3}, \mathrm{~L}_{4}, \ldots, \mathrm{L}_{23}, \mathrm{~L}_{21}$ d'autre part. On a préféré utiliser deux cernes âgés plutôt qu'un seul en guise de répétition. L'évolution de $r$ avec l'âge du cerne « jeune» montre que dès 9 ans, la liaison juvénile-adulte est significative. Elle semble atteindre un palier de 0,6 vers l'âge de 14-15 ans ; ce résultat est à rapprocher de l'apparition d'une certaine « cristallisation » des classements individuels avec l'âge.

Ces liaisons sont conformes à celles observées entre les circonférences 1956 et 1976, qui correspondent en gros aux fourchettes d'âges 1 à 10 ef 1 à 30 ans à partir de la moelle à la hauteur de prise de la carotte $(0,602 * *$ et $0,598 * *$ suivant que l'on raisonne ou non sur les arbres sélectionnés : voir tableau 4).

L'éclaircie réalisée en 1959 en faveur de la plupart des arbres sélectionnés (ceux d'Amance), c'est-à-dire en gros lorsque le cerne d'âge 13 (au sens défini par nous) s'est formé, s'est traduite par une amélioration de la croissance en circonférence (5,68 cm de 1959 à 1961 contre $5,19 \mathrm{~cm}$ de 1957 à 1959); en revanche, il est difficile d'en apprécier l'effet sur les corrélations juvénile-adulte au vu de la figure 3 .

Le problème des liaisons juvénile-adulte pour la largeur de cerne est controversée. Sur le Douglas, Reck et Sziklai (1973) ont trouvé sur des arbres dominants des coefficients significatifs et positifs au-delà des cinq premiers cernes ; en revanche, Thoby $(1975$, op. cit.) trouve des résultats différents : sur deux populations âgées de 60 ans environ, la liaison bois de 50 à 60 ans - bois juvénile pour la largeur reste 
nulle, que l'on considère dans leur ensemble les $1,2,3, \ldots$ ou 10 premiers cernes. Sur une autre provenance, la corrélation $r$ entre le bois des 6 à 10 premières années et celui formé de 20 à 30 ans est même négative. Comme le note cet auteur, ces résultats doivent être très liés aux conditions de traitement sylvicole (et sans doute aussi à l'échantillon étudié).

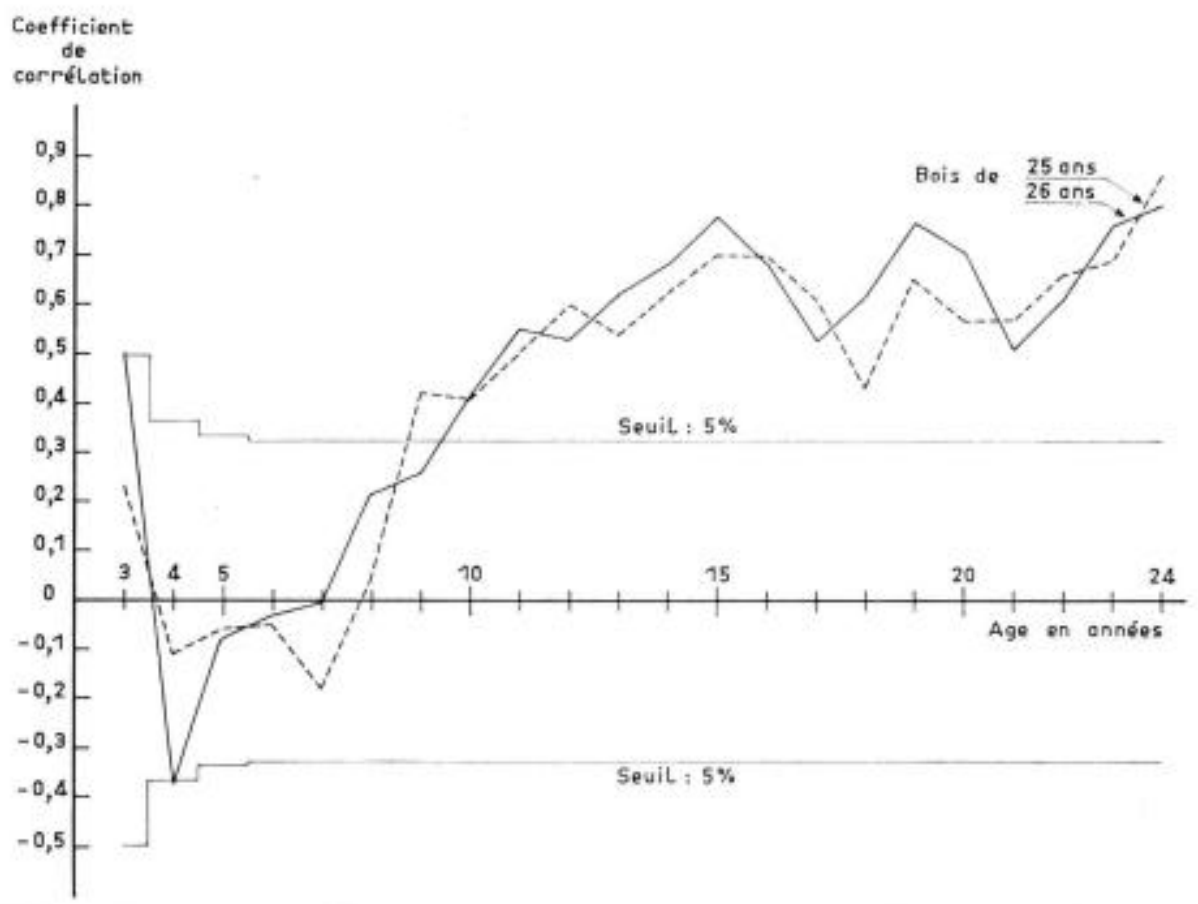

FIG. 3. - Liaisons juvénile-adulte pour la largeur de cerne suivant les âges. Arbres sélectionnés d'Amance et de La Sivrite.

- - liaisons bois de $3,4,5, \ldots, 24$ ans avec bois de 25 ans.

liaisons bois de $3,4,5, \ldots, 24$ ans avec bois de 26 ans.

Sevils de signification à 5 p. 100 :

-3 ans avec 25 et 26 ans : 0,497 ( $14 \mathrm{ddl}$ ).

-4 ans avec 25 et 26 ans : $0,367(27 \mathrm{ddl})$.

-5 ans avec 25 et 26 ans: 0,334 ( $33 \mathrm{ddl}$ ).

$-6,7, \ldots, 24$ ans avec 25 et 26 ans : 0,325 ( $35 \mathrm{ddl})$.

Juvenile-mature relationships for ring width according to age. Selected trees in Amance and La Sivrite.

2.14. Liaisons juvénile-adulte pour la densité.

La figure 4 présente les résultats exactement comme sur la figure 3. A la différence de la largeur de cerne, une bonne corrélation avec la densité du bois d'une vingtaine d'années se manifeste dès le $3^{e}$ cerne près de la moelle. Dès cet âge, le coefficient de corrélation « plafonne » vers 0,50 avec toutefois certaines valeurs non significatives.

Les résultats concordent avec ceux de Thoby (1975, op. cit.) sur Douglas qui note, pour deux des trois provenances étudiées, des corrélations significatives en prenant 


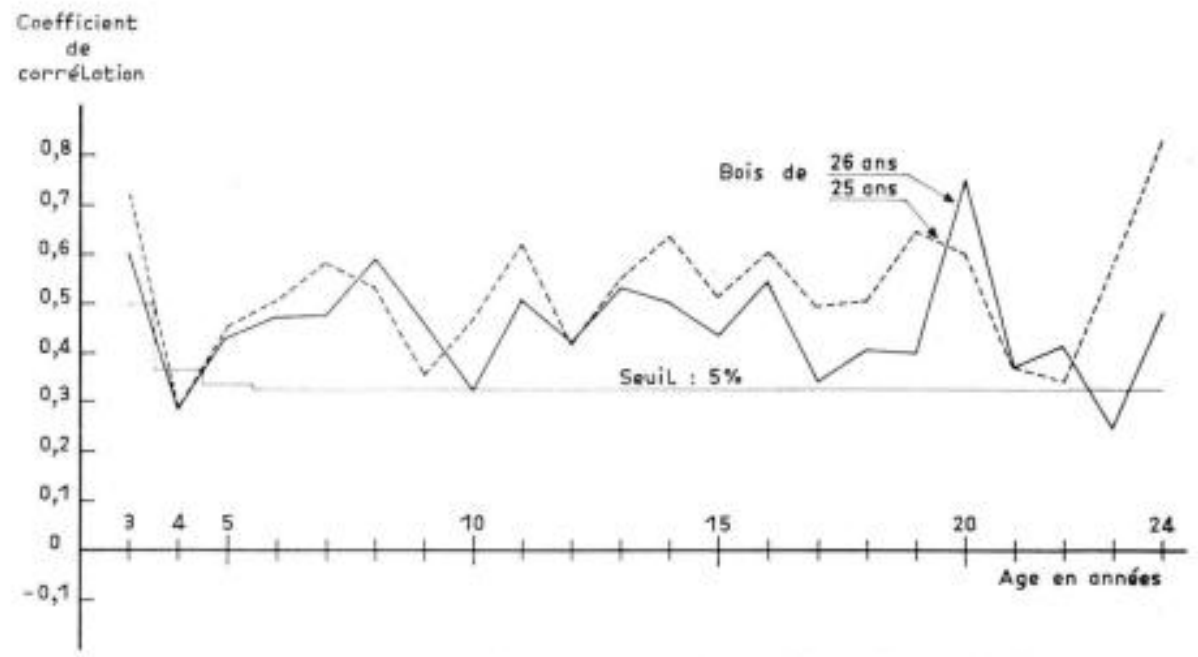

FIG. 4. - Lioisons juvénile-adulte pour la densité suivant les âges. Arbres sélectionnés d'Amance ef de La Sivrite.

Juvenile-mature relationships for ring wood density according to age. Selected trees in Amance and La Sivrite.

le cerne d'âge « un » pour le bois juvénile, ainsi que l'existence d'un palier à mesure que l'âge du bois jeune s'accroît. Le tableau 4 montre une corrélation très forte entre infradensités 1956 et 1976 ; elle dépasse sensiblement les valeurs que nous notons avec les densités de cerne. II peut y avoir plusieurs explications à ce phénomène :

- la mesure de l'infradensité est plus précise que celle de la densité,

- l'échantillon sur lequel on mesure l'infradensité 1956 est un tronçon de la carotte servant à obtenir l'infradensité 1976 : la liaison a donc un certain aspect « mécanique»,

- le fait de considérer plusieurs cernes (environ 10 et 30 respectivement pour les infradensités 1956 et 1976) atténue les fluctuations climatiques pouvant affecter la densité des cernes d'une année à l'autre ef qui doivent contribuer à diminuer les corrélations « âge par âge».

Ces deux arguments jouent également pour les comparaisons entre les corrélations circonférences 1956 et 1976 d'une part, $L_{n}\left(L_{n}<10\right.$ ans) et $L_{25}$ ou $L_{26}$ d'autre part. Le coefficient $r$ atteint 0,60 environ dans le premier cas, 0,40 dans le second (liaison entre cernes de 10 ans et de 25 ou 26 ans).

\subsection{Les corrélations canoniques juvénile-adulte.}

II peut fort bien arriver que l'information fournie par un seul accroissement annuel proche de la moelle ne soit pas suffisante pour en déduire le caractère du bois adulte, lui-même connu par un seul cerne. Ceci se traduit alors par des liaisons non significatives (voir fig. 3 et 4 ).

II n'est pas impossible cependant qu'en considérant une ou plusieurs caractéristiques d'un ou plusieurs cernes, à la fois côté moelle ef côté écorce, on puisse arriver à obtenir des corrélations différant statistiquement de zéro.

En ne prenant en comple que la largeur de cerne (ou la densité), on pourrait 
calculer côté moelle et côté écorce des moyennes sur plusieurs années. Cette méthode a été utilisée notamment par Thoby (1975, op. cit.) ; cependant, elle met sur un «pied d'égalité » les différents cernes ; on admet par exemple que celui d'âge 1 qui représente un volume très faible dans l'arbre « compte» autant qu'un autre d'âge plus élevé. Si l'on pense caractériser les cernes, non plus par la seule largeur (ou densité), mais par la largeur et la densité prises ensemble, ou même par une série de caractéristiques des cernes (composantes densitométriques par exemple), on voit que la méthode ci-dessus ne peut être employée.

L'analyse de corrélations canoniques fournit une méthode originale pour aborder le problème du déterminisme juvénile de la structure des accroissements annuels. Le principe en est rappelé par Bachacou et Berndt (1975) quí présentent en outre le programme ANACA (illustré d'un exemple) mis en place par eux à la Station de Biométrie du C.N.R.F. ; il permet d'estimer les liaisons entre deux groupes de variables dites « de gauche » et « de droite» (« juvéniles» et « adultes» pour le cas qui nous intéresse). En gros, il s'agit de chercher les deux combinaisons linéaires (des variables de droite/variables de gauche) qui présentent entre elles la plus forte corrélation. $\mathrm{Ce}$ sont les deux nouvelles variables obtenues appelées premières variables canoniques de gauche et de droite qui expriment la liaison linéaire optimale ( $p$ maxi) existant entre les deux groupes de variables. On peut ensuite déterminer de nouvelles variables canoniques (i.e. combinaisons linéaires de corrélation maximale) indépendantes de celles qui précèdent. Ainsi se révèlent successivement des liaisons indépendantes de corrélations décroissantes, dont le caractère significatif pourra être testé et dont l'interprétation pourra être facilitée par des descriptions graphiques des données.

Les corrélations cononiques observées sont testées à l'aide d'un test $x^{2}$. Le programme «ANACA » donne également les expressions des variables canoniques par rapport aux variables initiales et les saturations, c'est-à-dire les liaisons entre variables canoniques et variables de départ. Ces dernières sont très importantes car elles permettent d'interpréter les corrélations canoniques et de regarder dans quel sens interviennent les caractères initiaux.

Dans le cas particulier de cette éfude, on a travaillé sur les « résidus », c'est-àdire les écarts des valeurs individuelles à leur moyenne de famille (le programme ANACA ne prévoit pas en effet de liaisons au sens inter et intra). On obtient donc des corrélations proches des corrélations « intra » calculées plus haut.

II est important de souligner que les variables canoniques - de gauche ou de droite - sont des combinaisons linéaires des variables de départ. Le choix d'un modèle linéaire indique un certain a priori qui n'est toutefois pas trop audacieux lorsqu'il s'agit de variables de même caractère (largeur ou densité de cerne sur plusieurs années). II l'est davantage, surtout aux yeux du praticien qui s'intéresse au produit $\mathrm{D} \times \mathrm{L}$, lorsque, comme nous le verrons, nous nous pencherons sur une combinaison linéaire de ces deux variables.

On a pris successivement des nombres d'années croissants pour les variables « juvéniles» et « adultes», d'abord un an (cernes d'âges 5 et 26), puis 2 ans (cernes d'âges 5 et 6 d'une part, et 25 et 26 d'autre part), puis 3 ans (cernes d'âges $5,6,7$ et $24,25,26$ ) .... le premier cas étant à rapprocher des calculs effectués précédemment. On a considéré successivement la largeur seule, la densité seule et les deux ensemble. Afin d'alléger la présentation des résultats, seul «p maxi » a été donné. 
Les résultats résumés par la figure 5 montrent clairement une augmentation régulière de la corrélation canonique avec le nombre de cernes pris en compte, et ce pour les trois courbes, celle correspondant à la largeur et à la densité prises simultanément ayant en tous points une ordonnée supérieure aux deux autres. Pour la largeur, les valeurs deviennent significatives à partir de quatre cernes (âge 5 à 8 et 23 à 26), alors qu'elles le sont pour $D$ ainsi que pour $D$ et $L$ avec un seul cerne. La différence de comportement entre $D$ et $L$ n'est pas surprenante si l'on se reporte aux figures 3 et 4.

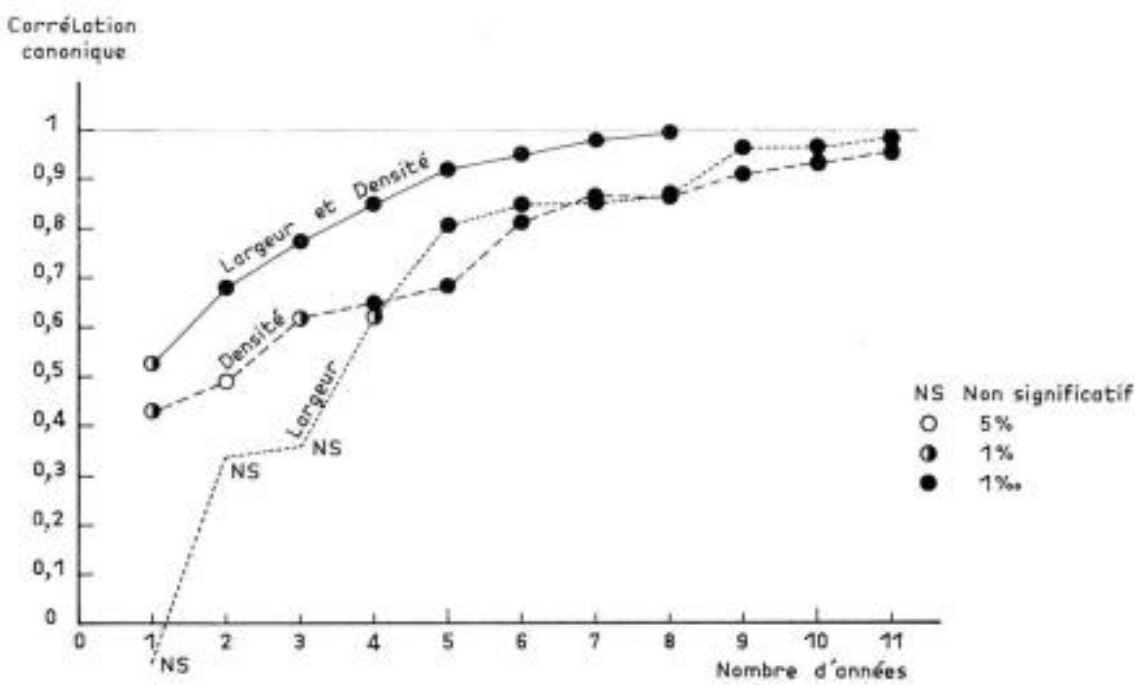

FIG. 5. - Evolution des corrélations canoniques avec le nombre d'annèes prises en comple (Nombre d'années à gauche $=$ nombre d'années à droife). Age minimum : 5 ans, âge maximum : 26 ans ; nombre d'individus : 43.

Remarque. - Les points d'abscisse 1 pour la densité ef la largeur correspondent à des corrélations simples.

Variation of cononic correlations according to the number of year (Number of years \& on the left $x=$ number of year $\approx$ on the right $x)$; minimum age : 5 years ; maximum age : 26 years; number on individuals : 43.

Remark. - The points with abscissa 1 for wood density and ring width correspond to correlations between two characteristics.

Dans les trois cas, les valeurs tendent vers 1 assez rapidement, plus vite pour $D+L$ que pour les deux autres très voisines dès que le nombre d'années dépasse 4. En prenant simultanément densité et largeur de 5 à 12 ans, il est possible de juger très exactement les caractéristiques du bois formé entre 18 et 26 ans (corrélations pratiquement égales à 1).

L'interprétation des variables canoniques est assez délicate. A titre d'exemple, on examinera le cas d'un nombre d'années égal à 4.

Pour la densité, les saturations sont positives ef très fortes (maximum 0,883, minimum 0,621) ; tous les cernes « jeunes» ont donc un même « poids», d'où l'intérêt d'une sélection précoce pour des valeurs fortes. Pour la largeur, les corrélations entre variables canoniques et variables initiales sont presque toutes négatives (sept cas sur 
huit) : en valeurs absolves, elles sont très différentes $(0,077$ à 0,950$)$. Si globalement cela ne contredit pas les résultats de la figure 3, certains âges contribuent donc plus que d'autres à « marquer» la ressemblance entre bois jeune et bois adulte du point de vue de ce caractère, d'où la possibilité d'interprétations biologiques intéressantes : un certain nombre d'arbres ayant formé leur bois de même âge la même année, il est possible que les corrélations fortes correspondent à des années de formation du bois jeune semblables climatiquement à celles de formation du bois adulte. C'est la première explication qui vient à l'esprit, mais il se peut également que certains âges aient un rôle particulier. Il serait bon alors de reprendre un travail de ce genre sur un autre matériel afin de savoir si le phénomène est reproductible. L'effet de la compétition serait également assez intéressant à considérer afin de nous apprendre si certains cernes, avant ou après éclaircie, marquent plus que d'autres la ressemblance jeune-adulte.

Enfin, en prenant en considération $D$ et $L$ simultanément, on observe des saturations fortes avec D, faibles pour L tant « d̀ droite » qu'« à gauche », ce qui démontre la contribution plus importante de la densité à la corrélation canonique. Ceci est conforme à l'allure des trois courbes de la figure 5 . On pourra regretter cependant de ne pas avoir disposé de cernes encore plus proches de la moelle qui auraient permis de juger de l'intérêt de sélections phénotypiques encore plus précoces que celles discutées ici.

On peut s'interroger pour conclure sur l'intérêt en l'espèce de l'utilisation de la méthode. Sur un plan très pratique, il est évident qu'il vaut mieux raisonner sur des caractères juvéniles ou adultes qui présentent une signification économique : densité, diamètre (voir tabl. 4), volume, masse de bois produite à un âge donné, ou bien si l'on dispose de $D$ et $L$ âge par âge en pondérant chaque cerne suivant le volume qu'il occupe dans l'arbre. On observe alors des corrélations qui peuvent guider le sylviculteur dans une opération d'éclaircie par exemple (pour la densité, ceci pose quelque problème technique). Pour le praticien, le vrai problème consiste en effet à estimer un caractère intéressant à retenir à l'âge adulte à partir du meilleur prédicteur juvénile qui peut d'ailleurs être multivariable.

L'analyse de corrélation canonique sert davantage à éfudier la structure de corrélation entre deux ensembles de variables, qui peuvent être de nature très différente à droite comme à gauche, et orienter éventuellement le choix de régressions simple ou multiple particulières (en vue de la prévision).

\section{2. - Liaisons juvénile-adulte pour l'infradensité (tabl. 5)}

La mesure de l'infradensité permettait d'étudier le bois situé au voisinage immédiat de la moelle ne pouvant être exploré par microdensitométrie.

Les carottes de sondage, qui avaient été radiographiées, ont été découpées, après repérage ef datation des cernes, en morceaux comptant quatre accroissements annuels ( 1 à 5 ans, 6 d̀ 9 ans, 10 à 13 ans, 14 à 17 ans, 18 à 21 ans, 22 à 25 ans, 26 à 29 ans).

Les corrélations sont relativement proches de celles observées dans la figure 4. Cependant, pour le bois de 1 à 5 ans, trois liaisons sont non significatives ef une significative d̀ 5 p. 100 seulement. 


\section{TABLEAU 5}

Liaisons juvénile-adulte pour l'infradensité suivant l'âge du bois.

Arbres sélectionnés d'Amance et de La Sivrife

Juvenile-mature relationships for wood basic densify in terms of oge.

Selected frees in Amance and La Sivrife

\begin{tabular}{|c|c|c|c|c|c|c|}
\hline & $\begin{array}{l}\text { Bois de } \\
6 \text { dे } 9 \text { ans }\end{array}$ & $\begin{array}{c}\text { Bois de } \\
10 \text { d } 13 \text { ans }\end{array}$ & $\begin{array}{c}\text { Bois de } \\
14 \text { d } 17 \text { ans }\end{array}$ & $\begin{array}{c}\text { Bois de } \\
18 \text { d̀ } 21 \text { ans }\end{array}$ & $\begin{array}{c}\text { Bois de } \\
22 \text { à } 25 \text { ans }\end{array}$ & $\begin{array}{c}\text { Bois de } \\
26 \text { d } 29 \text { ans }\end{array}$ \\
\hline Bois de 1 à 5 ans & $0,490 * *$ & $0,535 * *$ & $0,322 \mathrm{NS}$ & $0,130 \mathrm{NS}$ & $0,376 *$ & 0,306 NS \\
\hline \multicolumn{2}{|c|}{ Bois de 6 à 9 ans } & $0,489 *$ & $0,623 * *$ & $0,520 * *$ & $0,474 * *$ & 0,405 NS \\
\hline \multicolumn{3}{|c|}{ Bois de 10 a 13 ans } & $0,564 * *$ & $0,517 * *$ & $0,575 * *$ & $0,646 \cdots$ \\
\hline \multicolumn{4}{|c|}{ Bois de 14 d 17 ans } & $0,673 * *$ & $0,647 * *$ & $0,557 *$ \\
\hline \multicolumn{5}{|c|}{ Bois de 18 à 21 ans } & $0,614 \cdots$ & $0,736 * *$ \\
\hline \multicolumn{6}{|c|}{ Bois de 22 d 25 ans } & $0,805 * *$ \\
\hline
\end{tabular}

Nombre de degrés

\begin{tabular}{|c|c|c|c|c|c|}
\hline \multirow[t]{2}{*}{31} & 31 & 31 & 31 & 28 & 13 \\
\hline & 32 & 32 & 32 & 29 & 14 \\
\hline & & 32 & 32 & 29 & 14 \\
\hline & & & 32 & 29 & 14 \\
\hline \multicolumn{4}{|c|}{ degrés } & 29 & 14 \\
\hline \multicolumn{4}{|c|}{ rté des corrélations } & & 13 \\
\hline
\end{tabular}

NS : non significatif.

: significatif d 5 p. 100.

** significatif à 1 p. 100 .

de liberté des corrélations

Ceci est sans doute dû au fait que sont pris en compte ici les cernes d'âges 1 et 2 qui n'étaient pas représentés dans les calculs faisant intervenir la densité de cerne (le premier accroissement observé avait 3 ans); ceci est d'ailleurs confirmé par les travaux de Reck et Sziklai (1973, op. cit.) et Thoby (1975, op. cit.) sur le Douglas, qui trouvent des corrélations juvénile-adulte supérieures en supprimant le bois des trois ou quatre premiers cernes.

\section{3. - Liaisons entre d'autres caractères}

Elles sont présentées dans le tableau 6 qu'il faut rapprocher du tableau 4. Pour les critères auxométriques, seuls les accroissements courants ont été mentionnés.

On peut remarquer les corrélations très fortes entre les notes de débourrement d'une année à l'autre, entre les infradensités 1956 à 1976 ef également entre les accroissements courants sur la circonférence. Entre les accroissements en hauteur, aucun coefficient n'est significatif, ce qui est étonnant.

Aucune liaison défavorable infradensité-débourrement végétatif n'apparaît ; en revanche, la tendance de la corrélation infradensité - accroissement en circonférence est assez nettement négative, bien que rarement significative. Toutefois, avec les 
TABLEAU 6

Corrélations intrafamilles. Arbres sélectionnés d'Amance

Introprogeny correlations. Selected trees in Amonce

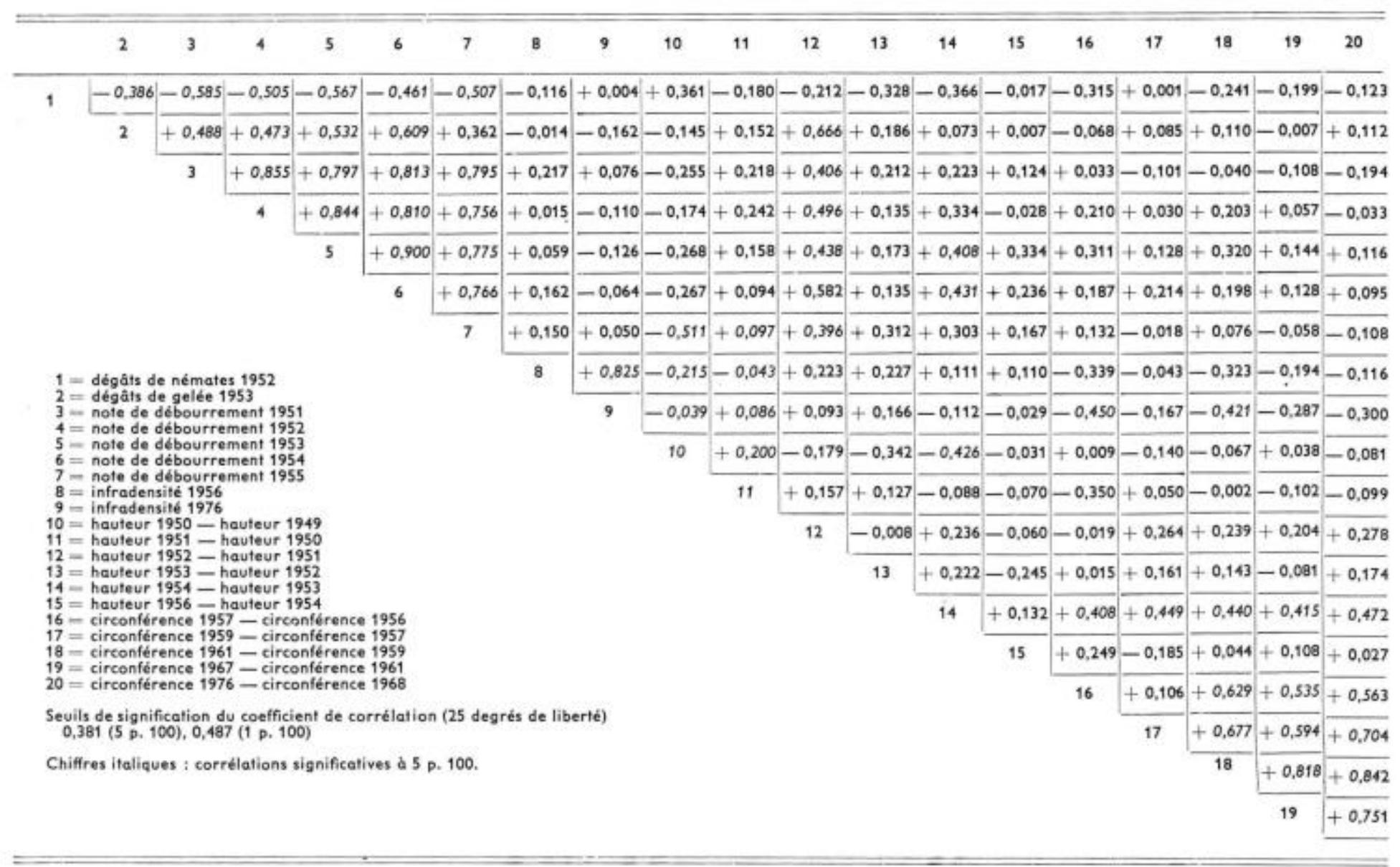


variables cumulées (circonférence 1956 et 1976 du tableau 4), cette liaison apparaît franchement défavorable.

II faut noter également des coefficients positifs entre dégâts de gelée 1953 et note de débourrement (ce qui est logique : plus l'arbre est précoce, plus les dégâts sont importants) et négatifs entre dégâts de némates et débourrement : les larves attaquent plus volontiers les arbres tardifs. Le phénomène est assez délicat à interpréter. Dans le Nord-Est de la France (CTGREF, 1975), les adultes émergent du sol après leur métamorphose vers la fin avril et pondent quasi immédiatement dans les jeunes aiguilles venant de se libérer des écailles du bourgeon. L'éclosion a lieu rapidement et les « fausses chenilles» s'alimentent sur les aiguilles de l'année du début mai à la mi-juin avant de tomber à terre, de s'enfouir et de s'entourer d'un cocon. Les arbres précoces ayant débourré suffisamment tôt auraient leurs aiguilles assez lignifiées pour n'être plus appétents. Ces résultats confirment les observations faites antérieurement sur une plantation comparative de provenances d'Epicéa (Bouvarel et Lemoine, 1957).

Ces considérations peuvent expliquer les excellentes liaisons entre l'accroissement en hauteur 1952 (année de forte attaque de némates) et toutes les notes de débourrement. Le fait que les corrélations n'apparaissent plus aussi nettement avec les pousses des années suivantes (sans attaque de némates) indique bien que les arbres débourrant tôt poussent plus vite que les sujets tardifs du fait qu'ils intéressent moins que les autres les ravageurs et non pas parce que leur saison de végétation commence plus tôt.

\section{Conclusion}

Malgré la médiocre qualité statistique du dispositif étudié qui nous a interdit une décomposition de la covariance phénotypique, ce travail a apporté des informations intéressantes.

Un apport original a été constitué par l'utilisation des corrélations canoniques : la prise en compte de plusieurs accroissements au voisinage du cœur et de l'écorce a permis de mettre en évidence pour la densité et la largeur de cernes des liaisons juvénile-adulte assez fortes pour les deux caractères, qui sont la marque d'un déterminisme assez précoce. Ceci n'apparaissait pas aussi nettement lorsqu'on considérait les corrélations classiques sur la largeur d'accroissement, y compris en prenant plusieurs cernes « jeunes» et « adultes». Le problème de l'interprétation des variables canoniques « jeune » et « adulte » reste cependant difficile pour la largeur de cerne.

Certains autres résultats méritent d'être soulignés :

- la sélection phénotypique individuelle précoce pour la densité semble possible, d̀ l'issue de quelques années de plantation. Elle pourrait intervenir à l'occasion des éclaircies à condition toutefois de disposer d'une méthodologie très simple d'évaluation (la mesure du couple de torsion n'est pas très adaptée sur des tiges de faible diamètre) :

- la liaison phénotypique débourrement - densité ne semble pas défavorable ; il en va différemment pour la liaison densité - vigueur. Pour ce dernier couple de caractères, des recherches permettant de chiffrer l'impact économique de la sélection pour l'un ou l'autre des caractères sont indispensables : 
- enfin, I'antinomie tardiveté du débourrement végétatif, sensibilité aux mémates semble difficile à contourner ; il faudrait pouvoir vérifier s'il existe des individus échappant à cette loi, et si leur résistance peut être liée à un certain profil biochimique (monoterpènes par exemple). Là encore, des recherches complémentaires semblent nécessaires ; l'importance des dégâts de némates dans les plantations d'Epicéa à basse altitude le justifierait.

$$
\text { Reçu pour publication en juillet } 1978 .
$$

\section{Summary}

\section{Phenofypic juvenile-mature correlations for wood densify and vigor in Norway spruce}

Following an extensive bibliographical review of juvenile-matur correlations for various characteristics in Norway spruce, the authors calculated these relationships in a 30 year-old stand. The characteristtes studied were ring width and wood density obtained by meat. of microdensitometry.

Correlations between juvenile and old rings were studied at various juvenile stages; unlike diameter growth, and considering one ring only, prediction of wood density seems possible at an early juvenile stage.

The canonical analysis was applied to the study of relationships between groups of juvenile and old rings. The advantages of this method with respect to usual correlations are discussed.

The unfavorable relationship between wood density and vigor is confirmed but the antagonism between the lateness of flushing and the high level of wood density is not verified.

It seems that the latest flushing individuals also are the most sensitive to Pristiphora abietina.

\section{Zusammenfassung}

\section{Die phenotypischen Korrelationen zwischen Jugend- und Reifestadium} für die Holzdichte und Wuchskraft bei der Fichte

Nach einer umfangreichen Literaturübersicht über die Korrelationen zwischen Jugend- und Reifestadium für verschiedene Merkmale bei der Fichte, haben die Autoren diese Beziehungen in einem um dreissig Jahre alten Bestand bestimmt, indem sie die durch mikrodensitometrische Messungen einzeln für jeden Jahrring gewonnenen Breiten und Dichten berücksichtigen.

Die Korrelationen zwischen den jungen und reifen Jahrringen wurden in Bezug auf diese beiden Merkmale unfersucht bei. Betrachtung von Ringen im unterschiedlichen Alter : daraus geht hervor, dass im Gegensatz zu dem Durchmesserzuwachs, die Dichte schon im jugendlichen Alter bei Untersuchung eines einzigen Rings im voraus bestimmt werden kann.

Die Analyse der kanonischen Korrelationen wurde bei Anwendung von Mengen junger und reifer Jahrringe durchgeführt. Der Vorteil der Methode gegenüber den klassischen Korrelationen wird diskutiert.

Die gewöhnlich beobachtete ungünstige Beziehung Dichte-Wuchskraft wird bestätigt. Dagegen ist der Antagonismus Austreiben-Dichte nicht festzustellen. abietina.

Offensichtlich sind die spatesten Individuen die empfindlichsten gegen den Befall von Pristiphora

\section{Streszczenie}

\section{Korelacje fenofypowe u šwierka pomiędzy gęstościg}

drewna i wzrastaniem u młodych drzewek, a gęsfościg̨ drewna i wzrastaniem

$$
\text { drzew starych }
$$

Po przejrzeniu obszernej bibliografii na temat korelacji między młodymi a starszymi drzewami, w odniesieniu do różnych cech świerka, autorzy oszacowali te zwigzzki dla frzydziestoletniego drzewostanu, rozpatrujaç szerokos̀ci i gęstos̀ci, otrzymane przy pomocy mikrogestościomierza, przez pomiar każdego stoju z osobna. 
Korelacje między młodymi a starszymi słojami były badane dla tych dwठch cech, przy jednoczesnym zróznicowaniu wieku młodych słoji ; z badań wynika, że przewidzenie gęstości drewna już w młodym wieku, na podstawie jednego tylko słoju. jest moźliwe, w przeciwiehistwie do przyrostu na szerokošc.

Zastosowanie metody korelacji kanonicznych zostało zrealizowane przez użycie zbiorów słoji młodych i starszych. Aktualnie analizowane sq̨ korzys̀ci wynikajgce $z$ użycia tej metody, w miejsce dotychezasowych korelacji klasycznych.

Niekorzystny zwig̨zek między gęstościg drewna a tempem wzrostu, zaobserwowany we wcześ-

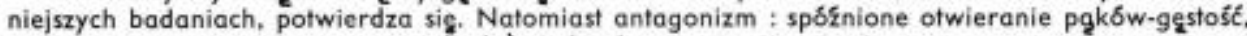
przy zastosowaniu naszego materiału doświadczalnego, nie ujawnia sic.

Wydaje się, że drzewa otwierajg̨ce pg̨ki najpరీ́niej sq̨ jednoczešnie najbardziej wraźliwe no ataki Pristiphoro abietina.

(traduction M. KACZMARSKA-NEPVEU)

\section{Références bibliographiques}

BACHACOU J., BERNDT L., 1975. Analyse des corrélations canoniques. Document d̀ distribution limitée. Station de Biométrie du C.N.R.F., n $n^{0} 1975 / 16$.

BADAN R., JEANTET G., 1975. A propos de la croissance juvénile de l'épicéa et de son amélioration par voie de sélection. La Forêt, 28 (9), 254-266.

BALDWIN H. I., ELIASON E. J., CARLSON D. E., 1973. IUFRO Norway Spruce provenance tests in New Hampshire and New York. Silvae genetica, 22 (4), 93-114.

BORNEBUSCH C., 1935. Proveniensforsög med Rödgran. Forstl. Forsögsw. Danmark, 13, $325-378$.

BOUVAREL P., 1962. Influence de l'origine des graines d'Epicéa sur la croissance en pépinière, la précocité et la fréquence des pousses d'août. Ann. Ec. Eaux et Forêts, Nancy, 19, 3, $415-439$.

BOUVAREL P., LEMOINE M., 1957. L'expérience internationale sur les provenances d'Epicéa (Picea excelsa Link.). Silvae Genetica, 6, 3/4, 91-97.

C.T.G.R.E.F., 1975. Information technique pour la surveillance et la protection phytosanitaire de la Forêt. Fascicule II.

DIETRICHSON J., 1968. Climatic damage, growth rhythm and height development : results from a 12 years old Norway spruce provenance trial at Rodser in Aremark. Medd. Norske Skogforsoksv. 25, 2, n० 88.

ERICSSON B., 1960. Studies of the genetic a wood density variation in Scotch pine and Norway spruce. Forest Research Institute of Sweden, report $n^{\circ} 4$.

FABRICIUS L., 1931. Pflanzenauslese in Saatbeet. Forstw. Cbl., 53, 449-457.

GISLERUD O., 1973. A wood density study in Norway spruce. Poper to the IUFRO joint Workshop ond Symposium on Norway Spruce provenances, Norway.

HEIKINHEIMO O., 1949. Toloksia kuusen ja männyn maantietsellisillä roduilla suorifetuista kokeista. Comm. Inst. for fenn., 37, 2, 44 p.

JANIN G., 1973. Détermination des diagrammes polaires des rendements et des indices de permanganate sur carottes droites et obliques. Essais de microclassage sur carottes obliques. Document à distribution limitée Station de Recherches sur la Qualité des Bois du C.N.R.F., no 1973/4.

KEYLWERTH R., 1954. Ein Beitrag zur qualitativer Zuwachs analyse. Holz als Roh u. Werkstoff, vol. 12, no 3, pp. 77-83.

KIELLANDER C. L., 1951. Sortskillnader i 10-ariga fälfförsök med svend och mellaneuropeisk gran. Svensk. Skogsw. Tidsk., 49, 3, 282-301.

KLEINSCHMIT R., 1961. Experiments with Spruce cuttings for a genetic test. Silvae genetica (10). 1 , 10-20.

KOWALSKI M., WLOCZEWSKI T., 1972. The height increment of the progeny of P, abies from different parts of Poland. Zeszyty noukowe Szkoly Glownej Gospodarstwa Wiejskiego w Warszawie, Lesnictwo, no 17, 7-19.

LACAZE J. F., 1969. Heritability of flushing in a Spruce population on the second plateau of the French Jura. Pap. 2 nd FAO/UFRO World Consulf. For. Tree Breed., Washington 1969, no FO. FTB $69,6 / 13$, P. 9 .

LACAZE J. F., 1970. Analyse d'une expérience multistationnelle de provenances d'Epicéa (Piceo abies Karst.). Ann. Sci. For, 27. (1), 5-37.

LACAZE J. F., POLGE H., 1970. Relations phénotypiques au stade juvénile entre la densité du bois et divers caractères phénologiques et de vigueur chez Picea abies. Karst. Ann. Sci. For.. 27 (3), 231-242.

LACAZE J. F., ARBEZ M., 1971. Variabilité infraspécifique de l'Epicéa. Héritabilité et corrélations génétiques de quelques caractères au stade juvénile. Ann. Sci. For., 28 (2), 141-183. 
LEWIS A. B., LINES R., 1973. Flushing time for Norway spruce. A comparison between assessments in the nursery and forest stages. Research and Development Paper, Forestry Commission UK, $n^{\circ} 101,5$ pp.

MERGEN F., BURLEY J., YEATMAN C. W., 1964. Variation in growth characteristics and wood properties of Norway Spruce. Tappi, 47 (8).

MUNCH E., 1949. Beiträge zur Forstpflanzenzüchtung. Bayer. Landw. G.m.b.H., München, 118 p.

NANSON A., 1968. La valeur des tests précoces dans la sélection des arbres forestiers en particulier au point de vue de la croissance. Dissert. Doctorat Gembloux, Stat. Rech. Eaux ef Forêts Groenendaal, H. S., nं 38,242 pp.

NANSON A., 1971. Tests de descendances d'Epicéa commun. Trav. Stat. Rech. Groenendaal, E., $n^{0} 4,64 \mathrm{p}$.

NEPVEU G., 1976. Les corrélations juvénile-adulte en matière d'étude de la qualité du bois. Document d̀ distribution limitée, Station de Recherches sur la Qualité des Bois du C.N.R.F., nº 1976/3.

NILSSON B., 1938. Om sambandet mellan moderträd och avkomma hos tal och gran. Svensk. Skogsv. Tidsk., 56, 1, 55-68.

PARDE J., 1977. Compte-rendu de mission au Canada (6 au 17/10/1977). Station de Sylviculture et de Production du C.N.R.F.

PETRACIC A., 1957. Prilog za poznavanje visinskog prirarta smrekovih (Picea abies Link.) stabalaca nostalih iz razlitico razvijenih sadnica iste sjetve. Glasn. Sumske Pokuse, 13, 5-17.

POLGE H., 1964. Le bois juvénile des conifères. Revue Forestière Française, $n^{\circ} 6,6$ 474-505.

POLGE H., 1966. Etablissement des courbes de variation de la densité du bois par exploration densitométrique de radiographies d'échantillons prélevés à la tarière de Pressler sur des arbres vivants. Applications dans les domaines technologique et physiologique. Thèse de Docteur ès-Sciences Appliquées, Faculté des Sciences de PUniversité de Nancy.

POLGE H., ILLY G., 1967. Observations sur I'anisotropie du Pin maritime des Landes. Ann. Sci. For. 24 (3), 205-231.

RECK S., 1972. Austreibeverhalten und Wuchseigenschaften bei Fichte aus einem FichtenKreuzungsversuch. Forstarchiv, 43 (4/5) 91-94.

RECK S., SZIKLAI O., 1973. Juvenile-mature correlation and wood characteristics of Douglas-fir. Meeting on provenance research in Douglas-fir, Göttingen, Sept. 3-6, IUFRO Working Party.

ROHMEDER E., 1952. Die Moglichkeiten einer Wachstumsteigerung bei den Baumarten Kiefer und Fichte durch das Massenausleseverfahren der fruhzeitigen Pflanzengrossensortierung. Z. Forstaen, 2, 22.

ROHMEDER E., MERZ W., SCHONBORN A. (von), 1962. Züchtung von gegen Intrustrieabgase resistenten Fichten und Kiefernsorten. Forstw. Cbl. 81, 11/12, 321-332.

ROHMEDER E., 1971. Die Züchtung der Fichte auf frühzeitige und starke Borkenbildung. Forstwiss. Cbl., 90 (2), 74-87.

RUBNER K., SCHONBACH H., 1957. Ergebnisse eines heute 20 jährigen Fichtenherkunftsversuches. I Teil - Die Fläche der Bayern - II Teil - Die Flächen in Thüringen und Sachsen. Silvae Genetica, 6, 3/4, 65-91.

TEISSIER DU CROS E., 1975. Premiers enseignements d'un test de descendances de Picea abies Karst des Vosges (Peuplement du Kertoff). Ann. Sci. For, 32 (3), 143-155.

THOBY M., 1975. Validité de tests précoces d'appréciation des caractères technologiques du bois de Douglas. Application à une plantation comparative de 24 provenances de Douglas (Peyrat le Chateau) en liaison avec des caractères morphologiques et auxométriques. Rapport de stage ENITEF, Station de Recherches sur la Qualité des Bois du C.N.R.F.

WEISGERBER H., DIETZE W., KLEINSCHMIT J., RACZ J., DIETERICH H., DIMPFLMEIER R., 1976. Ergebnisse des internationalen Fichten-Provenienzversuches 1962. I. Phenological observations and height growth up to the first evaluation in the field. Allgemeine Forst- und Jagdzeifung 147 (12), 227-235. 